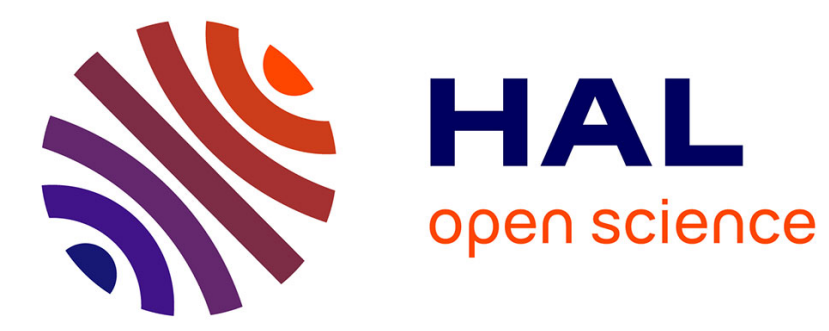

\title{
WiFi Dimensioning to offload LTE in 5G Networks
}

\author{
Danielle Saliba, Rodrigue Imad, Sébastien Houcke, Bachar El Hassan
}

\section{To cite this version:}

Danielle Saliba, Rodrigue Imad, Sébastien Houcke, Bachar El Hassan. WiFi Dimensioning to offload LTE in 5G Networks. CCWC 2019: 9th Annual Computing and Communication Workshop and Conference, Jan 2019, Las Vegas, United States. 10.1109/CCWC.2019.8666585 hal-02279869

\section{HAL Id: hal-02279869 https://hal.science/hal-02279869}

Submitted on 5 Sep 2019

HAL is a multi-disciplinary open access archive for the deposit and dissemination of scientific research documents, whether they are published or not. The documents may come from teaching and research institutions in France or abroad, or from public or private research centers.
L'archive ouverte pluridisciplinaire HAL, est destinée au dépôt et à la diffusion de documents scientifiques de niveau recherche, publiés ou non, émanant des établissements d'enseignement et de recherche français ou étrangers, des laboratoires publics ou privés. 


\section{WiFi Dimensioning to offload LTE in 5G Networks}

\author{
Danielle Saliba \\ Signal and Communication \\ Mines Telecom Atlantique \\ Brest, France \\ danielle.saliba@imt-atlantique.fr
}

\author{
Rodrigue Imad \\ Mechatronics \\ University of Balamand \\ $\mathrm{Al}$ Kurah, Lebanon \\ rodrigue.imad@balamand.edu.lb \\ Bachar El Hassan \\ Communication and Networking \\ Lebanese University \\ Tripoli, Lebanon \\ bachar_elhassan@ul.edu.lb
}

\author{
Sebastien Houcke \\ Signal and Communication \\ Mines Telecom Atlantique \\ Brest, France \\ sebastien.houcke@imt-atlantique.fr
}

\begin{abstract}
On the road towards 5G, a proliferation of Heterogeneous Networks (HetNets) is expected. Long Term Evolution (LTE) and Wireless Fidelity (WiFi) cooperation is needed in order to ensure a balanced traffic load based on different criteria so that the end user will benefit from the maximum throughput with no disturbance or deterioration in the service quality. Thus, alternative plans for exploiting already existing under-utilized $\mathrm{WiFi}$ infrastructure become more attractive than expanding the LTE spectrum or increasing the capacity by deployment of additional LTE Base Stations (BSs). To find a more effective spectrum utilization method, alleviate the spectrum scarcity problem of cellular networks and ensure additional capacity, we propose in this paper a solution to calculate the minimum needed number of WiFi Access Points (APs) that will be able to handle the transferred heavy users from LTE advanced (LTE-A) to WiFi. The dimensioning method that we propose in this paper is based on the remaining available capacity of $\mathrm{WiFi}$ channels taking into consideration the overlapping characteristics of the physical channels to estimate the percentage of busy time or occupation of the AP channels. Based on this approach, we can investigate first the remaining available capacity in terms of available throughput of $\mathrm{WiFi}$ that could be distributed over the transferred LTE users, then the minimum required number of WiFi APs that will be supporting the LTE network for efficient traffic offloading.
\end{abstract}

Keywords-5G, Heterogeneous Networks, LTE WiFi offload and coexistence, transmission power and throughput, WiFi Channels load estimation

\section{INTRODUCTION}

Data traffic over mobile networks is exhibiting an ongoing exponential growth due to the increasing number of smart phones and mobile broadband devices. This is expected to be increased exponentially with the deployment of the Internet of Things (IOT) till year 2020, and which necessitates a revolutionary approach to cope with the needed extra capacity.

In addition, there is a lack of available spectrum, and about $80 \%$ of mobile data traffic comes from indoor locations, and most of the current macro-cellular traffic comes from indoor or mobile users with fixed positions [1].

For those main reasons, the cooperation between LTE and WiFi will be an alleviating solution that will distribute the connected users efficiently between both cooperating networks and ensure throughput equilibration for the best Quality of Service and capacity limitations management.
WiFi could be considered as a good technology to support LTE systems in the $5 \mathrm{G}$ roadmap for many technical reasons. Basically, WiFi data rates are comparable with the ones of cellular networks, where it could achieve higher energy efficiency than the cellular networks. As well, in different WiFi networks, WiFi APs are installed easily and quickly in many residential areas and wide indoor environments with very affordable cost of investment and without any restrictions in the hardware size or needed physical or practical customization. We should also notice that most of smart devices are equipped with WiFi capabilities, in addition based on different studies, more than $80 \%$ of mobile traffic came from indoor environment. Thus, for all those reasons, WiFi could have advantage of establishing a communication infrastructure over other wireless communication networks [2].

However, most of current WiFi networks consist of randomly deployed WiFi cells since there is no limitations or policies on WiFi cell deployment [2]. The unplanned installation of WiFi APs may cause the WiFi networks to be implemented inefficiently.

There have been some studies on WiFi cell deployment problems. In [3], authors propose WiFi deployment algorithms based on realistic mobility characteristics of users to deploy WiFi APs for continuous service for mobile users based on maximum continuous coverage while WiFi network capacity was not considered. In [4], the number of APs required for WiFi offloading with different quality of service for data delivery was quantified. However, authors just provided a feasibility study on such offloading solution through real mobility traces and did not perform any mathematical analysis of this problem.

In [2], the minimum required number of WiFi APs was investigated based on the active users density, the coverage of the WiFi AP and the transmission probability of a user without taking into consideration the WiFi network available capacity.

In addition, many studies have analyzed the cooperation and offload between LTE and WiFi based on different criteria and assumptions. In [5], it was proposed to transfer WiFi users to the LTE system according to the availability of Channel State Information (CSI): the random transfer, the distance-based transfer, and the CSI-based transfer. In [1], the offload to WiFi was analyzed based on the Remaining Throughput Scheme (RTS) for Wi-Fi selection. In [6], the offload was analyzed based on the energy cost incurred to 
the cellular base stations and according to a routing policy within the overlay network.

As shown, it is obvious that cellular traffic load is partially offloaded if we use both LTE and WiFi together. However, the key issue is to quantify the WiFi APs needed to support a proper number of users while guaranteeing a suitable user experience requirements.

It is obvious that, a WiFi network will achieve higher throughput as more and more APs are installed. However, it is not a good solution if we consider an unlimited increase in the related capital and operational expenditure (CAPEX/OPEX) to install additional WiFi APs. Therefore, it is important to investigate the minimum required number of those APs that achieves a certain level of performance requirement.

In our paper, we propose a novel method in dimensioning the WiFi network to support certain heavy transferred users from LTE to WiFi based on the calculated remaining capacity of the WiFi overlapped physical channels.

In our scenario, the existing WiFi network constituted of minimum one AP will be handling the LTE offloaded traffic on top of its initial traffic, and then any needed extra capacity will be reflected by incrementing the number of WiFi APs.

This solution will alleviate the LTE eNB energy consumption for the heavy users, and thus instead of increasing the capacity and number of eNBs in the LTE network, we are proposing to increase the number of $\mathrm{WiFi}$ APs.

This architecture can provide a low-cost solution instead of other solutions such as increasing the number of LTE base stations or small cells that necessitate additional cost of investment.

In this case the investment in hardware, implementation and maintenance cost CAPEX and OPEX will be reduced, to note that these figures will not be discussed in this paper, however they will be elaborated in future works.

The rest of this paper is organized as follows. In section II, the system model along with the users transfer schemes are described. We calculate the available capacity of the WiFi network and dimension the needed number of WiFi APs in Section III. Section IV shows the numerical results describing the performance of the proposed solution. Section $\mathrm{V}$ summarizes the future related works and VI concludes the paper.

\section{System Model AND PROBlEM FoRMULATION}

We consider a network where an LTE advanced Base Station (BS) cell, also known as eNB, is covered by ' $\mathrm{K}$ ' WiFi APs (' $K$ ' unknown to be calculated) that will support the transfer of heavy users from LTE-A to WiFi where a sufficient capacity and proper $\mathrm{WiFi}$ coverage are available.

The proposed architecture is depicted in "Fig.1" where the eNB serves a set of Mobile Users (MUs) (or User Equipments (UEs)) that also have WiFi interfaces.

The proposed macrocell overlaps with a WiFi network, where the mobile users are in range with one or more WiFi APs. The amount of data to be downloaded or uploaded from/to the internet differe between different users, as well for their channel conditions with the base station.

As mentioned, our algorithm depends on the estimated channels load of the physical layer of WiFi network, where an accurate estimation of the channel occupation has been calculated [7].

Therefore, the offloading decision will not be random or based on the probability of WiFi channels occupation or on the Channel State Information (CSI). Instead, it will be based on the exact information sent by the WiFi network informing the LTE eNB about its remaining capacity.

The selection of the transferred users will not be random either. Instead, it will be based on the users with heavy data consumption depending on the requested throughput and transmitted power, so that we will calculate the minimum needed number of WiFi APs to cope with this number of transferred users.

Our framework is divided into two sequences to transfer cellular data traffic from LTE BS to WiFi:

- The first sequence is to determine the heavy users who will transmit the higher power and thus should be offloaded from the LTE system.

- The second sequence is WiFi remaining capacity calculation. This considered through WiFi APs dimensioning, and it is based on the remaining throughput of each WiFi AP.

We define the target average per user throughput that a WiFi network should assure for an efficient WiFi offloading. This average per user throughput is the criterion that based on it we will calculate the minimum required number of WiFi APs in the overlay network.

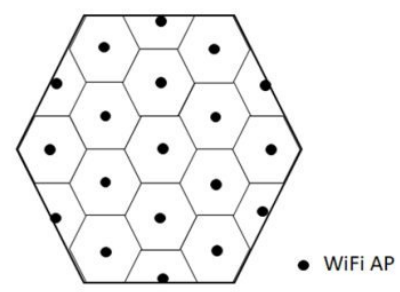

Fig. 1. An overlay network with 'K' WiFi APs deployment covering a regular hexagonal LTE-A cell. 
As illustrated in "Fig1", we consider a scenario with one LTE BS and ' $\mathrm{K}$ ' WiFi APs operating separately in licensed and unlicensed spectrum, respectively.

We further propose that we have a coverage area of 802.11n WiFi APs with no interference, each transmitting on an orthogonal channel in the $2.5 \mathrm{GHz}$ unlicensed spectrum selected based on the minimal calculated load value of the channels referring to the algorithm [7]. This model has also been adopted in other literatures, such as [2], [5].

The coexistence of WiFi and LTE could be facilitated by assuming that an inter-system coordinator exists, which performs the WiFi user transfer and resource allocation, as in [5]. We shall note that our proposal is very useful for the case where LTE-A and WiFi are deployed by the same network operator. In this case, the inter-system coordinator can be implemented by the cellular network operator itself. Otherwise, it can be implemented by a third-party vendor that provides service enhancement for both WiFi and LTE.

In our paper, the problem formulation for the LTE eNB is an energy minimization problem and not a throughput maximization problem. The energy minimization solution consists to identify the most heavy users who consume the most energy and require a high needed throughput, considered in our simulation greater or equal to $20 \mathrm{Mbps}$ [8]. This decision affects the capacity of the WiFi network, as the offloaded users should be in range with an AP having an adequate capacity.

\section{Proposed Dimensioning MethoD}

In this section, the proposed dimensioning method for the WiFi minimum needed number of APs ' $K$ ' is presented and discussed.

\section{A. LTE Heavy Users Selection}

To determine the heavy users in LTE that should be offloaded to WiFi network, the operator needs to determine the resource allocation policy, in terms of Resource Blocks (RBs) assignment and transmission power [6].

We consider the downlink operation of one LTE-A macro cellular base station for a time period of ' $\mathrm{T}$ ' subframes, possibly expanding over multiple frames.

There exists a set of ' $\mathrm{N}_{\mathrm{c}}$ ' users within the cell.

The base station has a set of ' $M$ ' available RBs that can be allocated to users in each subframe $(t=1,2, \ldots, T)$. The value of ' $M$ ' depends on the available spectrum. Hence, there are in total $\left(\mathrm{M}^{*} \mathrm{~T}\right) \mathrm{RBs}$. The system is considered quasistatic, i.e., users do not join or leave the cell during the current time period, and channels do not change significantly (flat fading). Note that, even if channels change rapidly, the eNB will not be aware of this fact, as users transmit their Channel Quality feedback Information (CQI) parameters only once during this time period.

In the beginning of the period, the eNB devises the RB assignment and power allocation policy for serving his users.

Let $\mathrm{x}_{\mathrm{nm}}(\mathrm{t}) \in\{0,1\}$ denote whether $\mathrm{RB}$ ' $\mathrm{m}$ ' $\epsilon$ ' $\mathrm{M}$ ' is

Identify applicable funding agency here. If none, delete this text box. allocated to user ' $n$ ' $\epsilon$ ' $N_{c}$ ' during subframe ' $t$ '.
Let $\mathrm{P}_{\mathrm{nm}}(\mathrm{t})$ denote the respective transmission power. For each RB, the base station can determine a different transmission power. However, the total power consumption should not exceed a maximum level of aggregated transmission power $\mathrm{P}_{\max }$ (Watt).

Assuming orthogonal allocation of RBs, and ignoring inter-cell interference, i.e., we assume that proper Enhanced Inter-Cell Interference Coordination (eICIC) techniques are applied, the instant rate for each user ' $n$ ' is calculated by [6]:

$$
r_{n}(t)=\sum_{m=1}^{M} x_{n m}(t) \cdot W_{b} \cdot \log \left(1+\frac{h_{n m} \cdot x_{n m}(t) \cdot P_{n m}(t)}{\sigma^{2}}\right)
$$

, where ' $\mathrm{W}_{\mathrm{b}}$ ' is the symbol rate per $\mathrm{RB}, \mathrm{h}_{\mathrm{nm}}$ the channel gain of user ' $\mathrm{n}$ ' in RB ' $\mathrm{m}$ ' during the current time period, $\sigma^{2}$ is a parameter considering the variance of the noise [9]. These parameters are estimated through the CQI feedback that is provided by the users, once every period ' $T$ '. Based on this policy, the operator determines which users will consume the most power and hence are most costly and should be transferred to $\mathrm{WiFi}$.

\section{B. Remaining WiFi Capacity}

To calculate the WiFi network remaining capacity, we need to measure the network load or occupation level.

The channels occupation in $\mathrm{WiFi}$ systems may be measured through the standard carrier sense mechanism Clear Channel Assessment (CCA). CCA is a physical carrier sense which listens to the received energy on the radio interface.

CCA is defined in the IEEE 802.11-2007 standards as part of the Physical Medium Dependent (PMD) and Physical Layer Convergence Protocol (PLCP) layer. Carrier sense refers the ability of an AP receiver to detect and decode an incoming WiFi signal preamble. CCA must be reported as BUSY when another WiFi signal preamble is detected, and must be held as BUSY for the length of the received frame as indicated in the frame's PLCP Length field. Typically, any incoming WiFi frame whose PLCP header can be decoded will cause CCA to report the medium as busy for the time required for the frame transmission to complete [10].

However instead of adopting the instant CCA info on each WiFi AP on the network to reflect the network occupation, we rely in this paper on the channel load estimation method previously analyzed in our paper [7], that enables us to scan and measure the occupation of all $\mathrm{WiFi}$ overlapped physical channels simultaneously, independently from the local measurement on each AP.

This estimation method will be facilitating the occupation measurements aggregation and processing time.

In addition, since initially this value is an instant occupation measure, we consider in this paper the average value of channels occupation during peak hours for several days within the LTE-WiFi HetNet, so the dimensioning calculations will be based on an averaged occupation value for several days to reflect more accurately the load of the WiFi network. 
Let ' $\alpha_{i}$ ' denotes the average load or occupation value of channel $\mathrm{i} ;\left(1-\alpha_{\mathrm{i}}\right)$ is therefore the available idle capacity of this WiFi channel.

In addition, since WiFi APs operate on the different 12 channels of the $802.11 \mathrm{n}$ system based on the minimum load value of the channel [7], different APs might be operating simultaneously on a specific channel ' $i$ ', taking into consideration that they are not neighbors APs to avoid the inter-channel interference. Therefore, the total available capacity of this channel ' $\mathrm{i}$ ' will be divided between at least two APs.

If we consider $t_{i}$ as the number of APs operating simultaneously under the different frequencies of the WiFi channels $\left(1<\mathrm{t}_{\mathrm{i}}<12\right)$, we can deduce the below equation:

$$
K=\sum_{i=1}^{12} t_{i}
$$

$K \geq 1$ is the number of WiFi APs to be calculated.

Consequently, we can define the available capacity in terms of bit rate for a WiFi $\mathrm{AP} L(L=1, \ldots, K)$, operating on a frequency of channel $i$, and for the whole WiFi network, denoted as $R_{L}$ and $R_{\text {tot }}$ respectively, as following:

$$
\begin{array}{r}
R_{L}=R_{w_{\max }} \frac{\left(1-\alpha_{i}\right)}{t_{i}} \\
R_{\text {tot }}=\sum_{I_{t}=1}^{K} R_{L}
\end{array}
$$

,where $R_{w_{\max }}$ is the maximum throughput of the WiFi APs (considered as same releases and specs), $i$ is the channel number of $\mathrm{WiFi}(i=1, \ldots, 12), R_{\text {tot }}$ is the total remaining capacity or throughput of the WiFi network and $\mathrm{R}_{\mathrm{L}}$ is the remaining capacity or throughput of the $\mathrm{WiFi} \mathrm{AP}$ $L(L=1, \ldots, K)$.

From equation (4), we can estimate the total available capacity of the WiFi network, and thus dimension the minimum needed number of WiFi APs that will handle the transferred LTE users according to certain throughput criteria that will be analyzed in the next section.

\section{Dimensioning of the WiFi Network}

To ensure the same user experience, the average per-user throughput offered by the WiFi network should be at least equal to or higher than the cellular network throughput.

Based on this constraint, we set the target average per-user WiFi throughput as follows [1]:

$$
S_{W}^{u s e r} \geq S_{C}^{u s e r}
$$

where $S_{W}^{\text {user }}$ and $S_{C}^{\text {user }}$ represent the average per-user WiFi throughput and the average per-user cellular throughput respectively. From equation (4) we can conclude the below equation:

$$
\operatorname{Avg}\left(S_{W}^{u s e r}\right)=\frac{R_{\text {tot }}}{N_{W}}
$$

$N w$ is the active number of heavy users calculated from equation (1) who are bypassing certain max LTE throughput considered in the simulations results as $20 \mathrm{Mbps}$ as average [8], and that will be transferred from LTE to WiFi.

While considering a maximum threshold within the LTE network, where each user exceeding this threshold should be transferred to WiFi, and while setting this threshold as the minimum needed capacity per user to be ensured by the WiFi network, we calculate the minimum required number of WiFi APs $K$ that achieves the target average per user WiFi throughput.

We can express the mathematical expression of $K$ by:

$$
K=\underset{K}{\operatorname{argmin}} S_{W}^{\text {user }}
$$

such that $\operatorname{Avg}\left(S_{W}^{u s e r}\right) \geq r_{n}(t)$ calculated in equation (1).

\section{SIMULATIONS RESULTS AND PERFORMANCE EVALUATION}

We consider an LTE FDD system for one eNB cell operating in $1800 \mathrm{MHz}$ with an available bandwidth of 10 $\mathrm{MHz}$ [6] [11], with a WiFi 802.11n network operating in 2.5 $\mathrm{GHz}$ bandwidth with 12 overlapped channels on the physical layer [7].

Every Transmission Time Interval (TTI), the eNB makes a scheduling decision to dynamically assign the available time-frequency RBs to the UEs. The eNB scheduler aims at power minimization while also at satisfying UEs demands. Table I summarizes the basic system model configuration,

while considering $N_{c}$ the total number of LTE users operating in the heterogeneous network varying from 10 to 100 users per eNB making simultaneously data session.

Based on the configured setup, we present numerical results by using MATLAB to analyze the minimum required number of WiFi APs versus LTE and WiFi throughput.

By varying the number of simultaneous active users in the LTE cell from 10 to 100 active users, "Fig. 2" represents the number of users considered as heavy users and that need to be offloaded to WiFi network.

TABLE I. SYSTEM CONFIGURATION

\begin{tabular}{|c|c|}
\hline Parameters & Values \\
\hline Bandwidth & $10 \mathrm{MHz}$ \\
\hline Duration & $10 \mathrm{~ms}$ \\
\hline RBs per Tslot & 50 \\
\hline RBs per TTI & 100 \\
\hline subcarriers per RB & 12 \\
\hline max eNB TX Power & $43 \mathrm{dBm}$ \\
\hline max UE TX Power & $23 \mathrm{dBm}$ \\
\hline Symbols per RB & 7 \\
\hline number of subframes T & 20 \\
\hline $\begin{array}{c}\text { Block Error Rate } \\
\text { requirement }\end{array}$ & 0.1 \\
\hline Channel Gain & $6 \mathrm{~dB}$ \\
\hline $\begin{array}{c}\text { Max capacity of WiFi } \\
\text { AP Rwmax }\end{array}$ & $600 \mathrm{Mbps}$ \\
\hline
\end{tabular}

Taking into consideration that the LTE users will be offloaded when their demands exceed the $20 \mathrm{Mbps}$, considered as the average per user throughput in LTE-A network [8], the minimum needed number of WiFi APs, and the acquired throughput in the WiFi network are shown in 
"Fig. 3" and "Fig. 4" respectively, noting that there is no restriction in this case on the maximum offered throughput per user in the WiFi network.

As we can observe, with few LTE users to be offloaded, the WiFi network with only one AP can provide up to around $120 \mathrm{Mbps}$ as theoretical value on top of its existing users. The WiFi throughput per user decreases with the additional number of offloaded simultaneous active users, with an average of $40 \mathrm{Mbps}$, thus greater than the maximum defined threshold in the LTE network (20 Mbps). By adopting this method, in addition to the saved cost when increasing the WiFi APs (to a maximum of 4 or 5 APs) in indoors environment instead of increasing the eNBs, the user experience will be enhanced instead of suffering from any possible congestion or throughput deterioration with limited number of LTE eNBs.

If we take the scenario of a restricted threshold of throughput offered to the offloaded users in the WiFi network (e.g. a max of $20 \mathrm{Mbps}$ ), the needed number of WiFi APs will be reduced as presented in "Fig. 5".

Finally, to pinpoint the saving in LTE, we have measured the average power consumption related to the transmitted power saved after being transferred to WiFi [12].

"Fig. 6" and "Fig. 7" represent the average power consumption saving in the eNB in Watts, and the percentage of power saving in respect to the total consumed power, respectively.

As we can observe, there is on average $40 \%$ saving of the total consumed power in the eNB. The power consumption saving is expected to grow when the number of offloaded users is increasing.

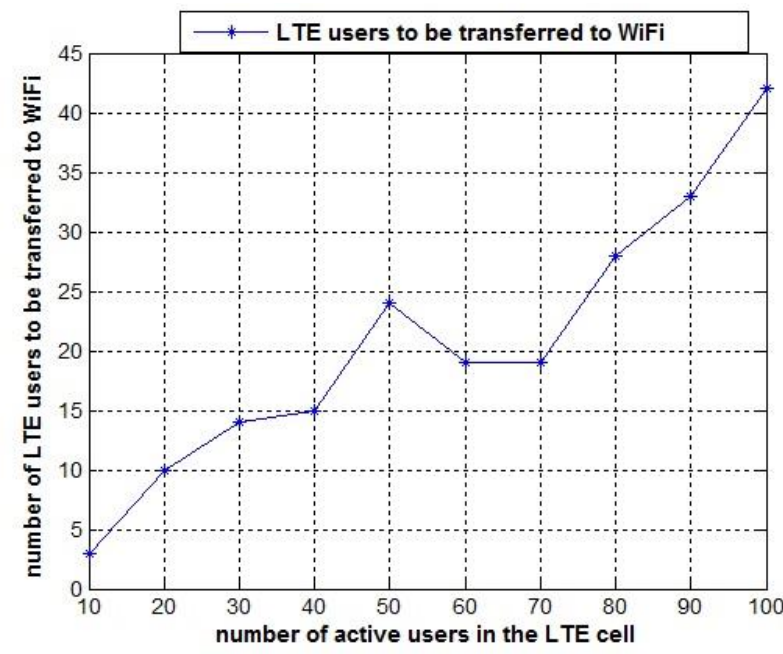

Fig. 2. Number of users to be offloaded to WiFi in respect to the total number of active users in the LTE cell.

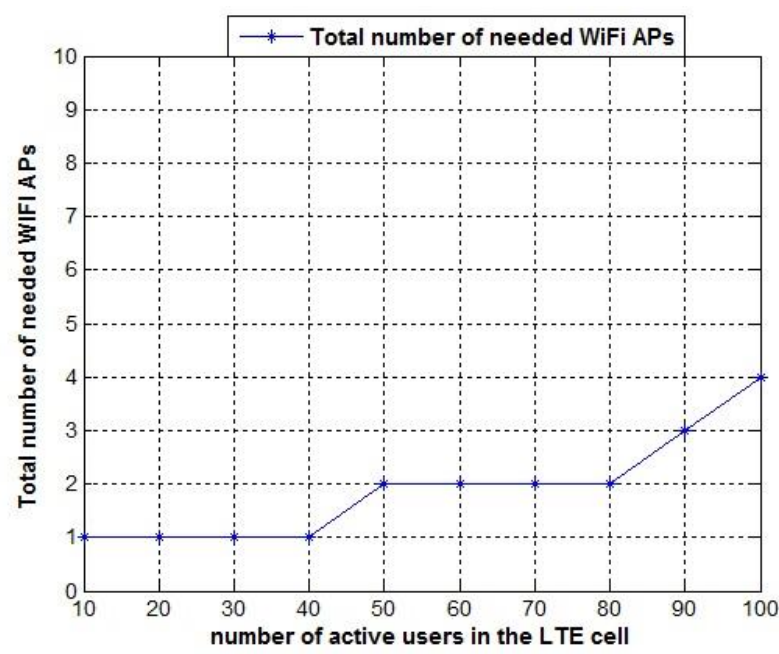

Fig. 3. Total number of needed WiFi APs with no limitation on average per user WiFi throughput.

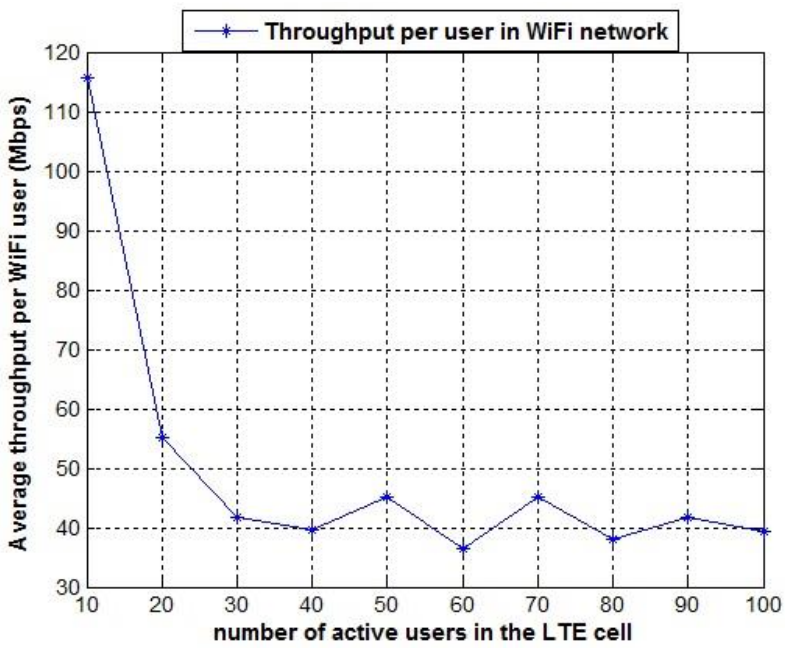

Fig. 4. Average per user WiFi throughput (Mbps).

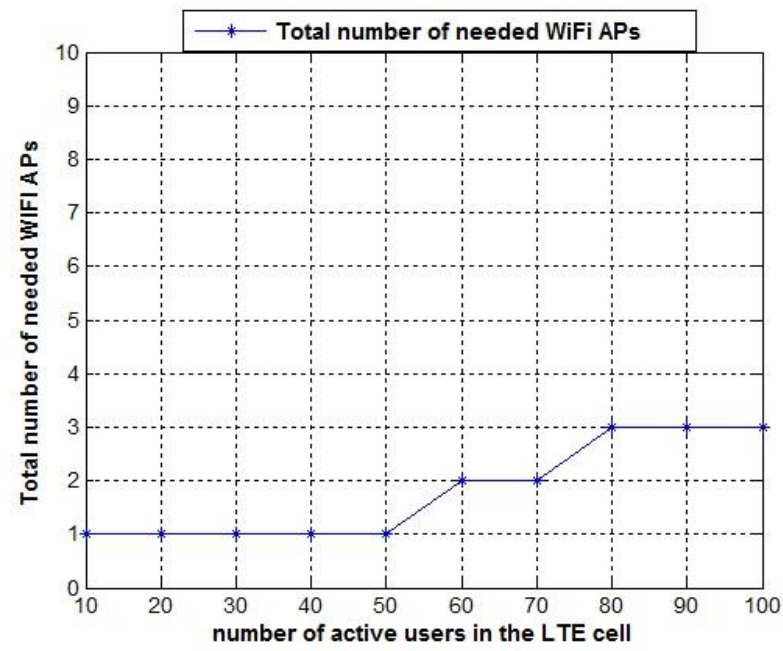

Fig. 5. Total number of needed WiFi APs with average per user $\mathrm{WiFi}$ throughput set to $20 \mathrm{Mbps}$ maximum. 


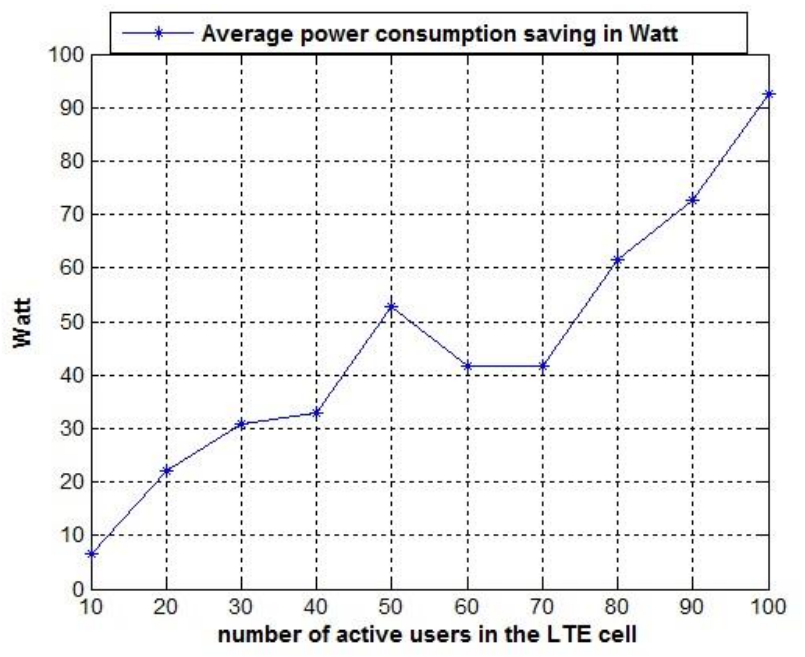

Fig. 6. Average Power consumption saving in Watt.

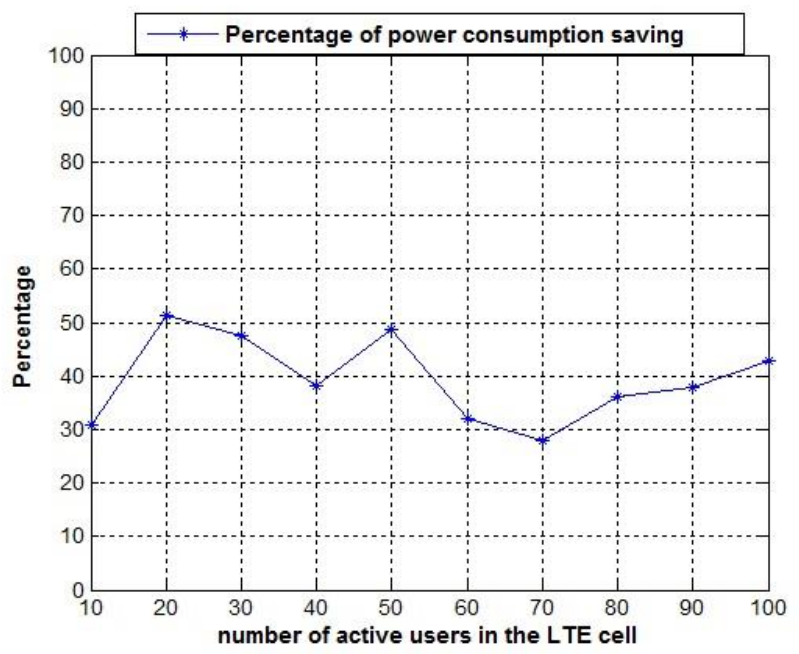

Fig. 7. Percentage of Power Consumption saving.

\section{FUTURE WORK}

Our Future work will entail to examine the distance criteria on top of our mathematical approach, in order to avoid the random transfer that might be overloading the WiFi Network without guaranteeing an acceptable Quality of Service for the end user.

This characteristic will be added so that if users are under the coverage of WiFi AP and are heavy LTE users so they will be offloaded to $\mathrm{WiFi}$, otherwise the user will remain connected to the LTE BS independently from his required data consumption.

As well, gaming theory techniques could be applied in order to estimate the benefit and cost saving in LTE and WiFi networks after applying the proposed technique of load balancing.

\section{CONCLUSION}

In this paper, we have proposed a mathematical approach to find the minimum required number of $\mathrm{WiFi}$ APs to support the heavy users' traffic transferred from LTE to WiFi network based on the remaining available capacity of the WiFi network.

This capacity was estimated taking into consideration the overlapping characteristics of the physical channels of the WiFi technology, where we can estimate the percentage of busy time and idle time of the channel.

Through the mathematical approach proposed in our paper to calculate the minimum required number of $\mathrm{WiFi}$ APs, we can ensure an efficient coexistence between LTE-A and WiFi HetNets, while providing a minimum acceptable bit rate to the end users, and with minimum required hardware and investment.

\section{REFERENCES}

[1] Ibrahim Elgendi, Kumudu S. Munasinghe, and Abbas Jamalipour, "Traffic Offloading for 5G: L-LTE or Wi-Fi," IEEE Conference on Computer Communications Workshops (INFOCOM WKSHPS): IECCO: Integrating Edge Computing, Caching, and Offloading in Next Generation Networks, 2017.

[2] JaYeong Kim, Nah-Oak Song, Byoung Hoon Jung, Hansung Leem, and Dan Keun Sung, "Placement of WiFi Access Points for Efficient WiFi Offloading in an Overlay Network," IEEE 24th International Symposium on Personal, Indoor and Mobile Radio Communications: Mobile and Wireless Networks, 2013.

[3] Tian Wang, Guoliang Xing, Minming Li, and Weijia Jia, "Efficient WiFi Deployment Algorithms based on Realistic Mobility Characteristics," IEEE MASS, 2010.

[4] Savio Dimatteo, Pan Hui, Bo Han, and Victor O.K. Li, "Cellular Traffic Offloading through WiFi Networks," IEEE MASS, 2011.

[5] Qimei Chen, Guanding Yu, Amine Maaref, Geoffrey Ye Li, and Aiping Huang, "Rethinking Mobile Data Offloading for LTE in Unlicensed Spectrum," IEEE Transactions on Wireless Communications, VOL. 15, NO. 7, July 2016.

[6] Apostolos Apostolaras, George Iosifidis, Kostas Chounos, Thanasis Korakis, Leandros Tassiulas, "C2M: Mobile Data Offloading to Mesh Networks," IEEE Global Communications Conference, 2014.

[7] Danielle Saliba, Rodrigue Imad, Sebastien Houcke, "Overlapped Physical Channels Load Measurement in 802.11 Networks," International Journal of Advanced Research in Computer Science, Sep 2017.

[8] Kishor Pratap Singh, Pradeep Kumar Chopra, "Throughput Computation of LTE-A Network for Urban Area," International Journal of Advanced Research in Electronics and Communication Engineering (IJARECE) Volume 3, Issue 11, Nov 2014.

[9] YuPeng Wang, Dongyao Wang, JiYong Pang, and Gang Shen, "Selfoptimization of Downlink Transmission Power in 3GPP LTE-A Heterogeneous Network," Vehicular Technology Conference (VTC Fall), IEEE Sep 2012.

[10] Andrew S. Tanenbaum and David J. Wetherall, "Computer Networks" Fifth Edition, Jan 2010

[11] Pierre Bertrand, "Channel Gain Estimation from Sounding Reference Signal in LTE," IEEE 73rd Vehicular Technology Conference (VTC Spring), 2011

[12] Bjoern Dusza, Christoph Ide, Liang Cheng and Christian Wietfeld, "An Accurate Measurement-Based Power Consumption Model for LTE Uplink Transmissions," Proc. of IEEE INFOCOM 2013. 